Bernd Schulte Ingo Schäfer Jens Reimer

\section{Platzierungskriterien in der Suchttherapie in Deutschland - ein Diskussionsvorschlag}

Patient Placement Criteria in Addiction Treatment in Germany - a Proposal

\section{Zusammenfassung}

In den USA haben sich die Patientenzuordnungskriterien der Amerikanischen Gesellschaft für Suchtmedizin (ASAM) in den letzten 10 Jahren zu einem nationalen Behandlungsmodell entwickelt und finden entsprechend häufig Anwendung in der Behandlung von Suchtpatienten mit und ohne (psychiatrische) Komorbidität.

Die Zuordnungskriterien stellen Richtlinien dar, um erwachsene oder jugendliche Patienten mit einem spezifischen Muster einer substanzbezogenen Erkrankung der für sie angemessensten Behandlung zuzuordnen. Nach Diagnosestellung und biopsychosozialer Beurteilung der Schwere der Erkrankung anhand von Bewertungsdimensionen werden die Patienten entsprechend in unterschiedlichen Versorgungsstufen behandelt.

Die Anforderungen an die Versorgung suchtkranker Patienten unterliegen ständigen Veränderungen und bedürfen multiprofessioneller therapeutischer Angebote. Verschiedene wissenschaftliche Studien bestätigen den ASAM-Kriterien eine gute Durchführbarkeit, Reliabilität und Validität und stützen die Relevanz zur Operationalisierung von Indikationskriterien. Die Implementierung von Patientenzuordnungskriterien in das deutsche Versorgungssystem könnte eine Chance bieten, Ressourcen patientenorientierter einzusetzen. Zugleich würden Veränderungen auf verschiedenen Ebenen des Behandlungs- und Versorgungssystems notwendig. Hilfe für eine mögliche Implementierung kann daher die Versorgungsforschung bieten.

\section{Schliuisselwörter}

Richtlinien · Versorgung · Ressourcen · Versorgungsforschung

\section{Abstract}

During the last ten years, placement criteria for patients as established by the American Society of Addiction Medicine (ASAM) have increasingly become a national treatment model in the United States and have, accordingly, been frequently applied in treatment of addicted patients with and without (psychiatric) comorbidity.

Placement criteria are guidelines for assigning adult and adolescent patients with specific patterns of substance-related illness to the treatment most suitable. After the diagnosis and biopsychosocial assessment of the severity of illness based on assessment scales, patients are treated in different settings according to the degree of severity.

Treatment standards for addicted patients are subject to constant changes and require multiprofessional treatment programmes. A number of studies confirm the feasibility, reliability and validity of the ASAM criteria and their relevance for the operationalisation of indication criteria. The implementation of placement criteria in the German health system could be an opportunity to use the available resources more effectively to benefit patients. Changes on various levels of the addiction and health care system would become necessary. The implementation could be assisted by health care research.

\section{Key words}

Guidelines · health care $\cdot$ resources $\cdot$ health care research Psychosoziale Medizin, Psychiatrie und Psychotherapie im UKE · Martinistr. 52 · 20246 Hamburg . E-mail: reimer@uke.uni-hamburg.de 
Einleitung

Die Patientenzuordnungskriterien der Amerikanischen Gesellschaft für Suchtmedizin (ASAM) zur Behandlung substanzbezogener Erkrankungen entsprechen klinischen Leitlinien, die das Ziel haben, mittels eines multidimensionalen Bewertungssystems eine optimale Passung zwischen den Entitäten Patient/Diagnosen einerseits und Behandlungsmodus/-intensität andererseits zu finden. Die Entwicklung der Patientenzuordnungskriterien lässt sich in den USA bis in die 80er-Jahre zurückverfolgen. Nach verschiedenen Versionen (u.a. Cleveland Criteria, National Association of Addiction Treatment Providers (NAATP) Criteria, NAATP-ASAMCriteria, ASAM-Patient Placement Critera -1 \& -2) sind im April 2001 die aktuell geltenden Kriterien, die „ASAM-Patient-Placement-Criteria-2-revised“, erschienen, die die Grundlage für diesen Artikel bieten [1]. Wie in allen medizinischen Versorgungsbereichen besteht auch in der Suchttherapie ein hoher Bedarf an Werkzeugen, die durch Effizienz und Kosteneffektivität zum einen eine hohe Qualität in der Versorgung gewährleisten und zum anderen durch einen verantwortungsvollen Umgang mit Ressourcen den Bestand des Versorgungssystems absichern helfen.

Im Folgenden werden die ASAM-Kriterien zur Behandlung von substanzbezogenen Krankheiten vorgestellt und Voraussetzungen für eine mögliche Implementierung in das deutsche Versorgungssystem diskutiert.

\section{Theoretische Grundlagen}

Die Besorgnis über das aufgrund von Über- und Unterversorgung ineffiziente und mit hohen Kosten belastete Versorgungssystem war einer der Gründe, der Ende der 80er-Jahre in den Vereinigten Staaten von Amerika zu neuen, nationalen Modellen in der medizinischen Versorgung geführt hat. $\mathrm{Zu}$ diesem Zeitpunkt bestand ein Mangel an adäquaten, speziellen Behandlungsebenen für Suchtpatienten. Viele Behandlungsprogramme sahen für die jeweilige Suchterkrankung Standardtherapien vor, die sich je nach den gesetzlichen Vorgaben (z. B. bezogen auf die Behandlungsdauer) der Bundesstaaten unterschieden. Der Gebrauch von spezifischen Behandlungsmodellen, wie den Patientenzuordnungskriterien, setzt einen Managed-Care-Prozess voraus, der die Qualität der Versorgung sowie deren Kosten bemessen kann. In den USA war Managed Care eine lange Zeit lang nur dem privaten Gesundheitssektor vorbehalten. Mit Hilfe der nationalen Gesundheitsreform konnten jedoch die Grenzen zwischen den privat finanzierten und öffentlich finanzierten Gesundheitssektoren geöffnet werden. Seither werden ManagedCare-Prinzipien auch im öffentlichen Sektor angewendet. In der Suchttherapie wurden ebenfalls entsprechende Ansätze zum Übergang in eine kosteneffektive Versorgung entwickelt. Im Gegensatz zu den öffentlich versicherten Patienten wurden die meisten privaten Patienten mit einer Suchterkrankung in einem 12-stufigen Behandlungsmodell für einen festen Zeitraum stationär behandelt, so dass man von einer Einheitsbehandlung sprechen konnte. Die Suchtbehandlung definierte sich vorher einzig aus der Diagnose mit entsprechend standardisierten Therapieansätzen. Zwar zeigten wissenschaftliche Studien, dass diese Behandlungsstrategien insgesamt zu einer Reduzierung des Drogenkonsums und zu einer Verbesserung der körper- lichen, sozialen und psychischen Gesundheit führten, doch waren die zur Verfügung gestellten Behandlungsangebote therapeutisch bei weitem nicht für jeden Patienten geeignet.

Heute steht beiden Versicherungssektoren in den Vereinigten Staaten von Amerika eine große Anzahl verschiedener Konzepte zur Behandlung von Suchterkrankungen zur Verfügung. Dementsprechend sind Kliniker gefordert, für ihre Patienten die angemessenste Behandlung auszuwählen. Zur Steigerung der Behandlungsqualität und zur effektiven Ressourcennutzung sind amerikanische Suchtmediziner zu einem System der Patientenzuordnung in Behandlungsstufen übergegangen, das mit einer Bandbreite von Behandlungsmodalitäten und einem genauen $\mathrm{Zu}$ schnitt auf die Bedürfnisse des Patienten eine optimale Versorgung gewährleistet. Ein solches System der Patientenzuordnung in Behandlungsstufen ist sowohl auf den Schweregrad der Abhängigkeitserkrankung als auch auf die Diagnose ausgerichtet. Die Diagnose ist somit eine hilfreiche, jedoch nicht mehr alleinige Determinante der Behandlung. Und dennoch hängt der Erfolg einer klinischen Behandlung von der richtig gestellten Diagnose ab. Aus diesem Grund orientieren sich die ASAM-Kriterien in der Diagnosestellung an allgemein gültigen Manualen wie den „Diagnostic and Statistical Manual of Mental Disorders“ (DSM IV) der amerikanischen Gesellschaft für Psychiatrie.

Da eine Vielzahl von Faktoren Einfluss auf Suchterkrankungen haben, ist eine rein medizinische Bewertung für die Diagnosestellung aber nicht ausreichend. Bereits Ende der 80er-Jahre forderten Donovan und Wallace die Integration der medizinischen Diagnose in ein biopsychosoziales Modell $[2,3]$. Sucht ist demnach in Ursprung, Ausdruck und Behandlung als eine biopsychosoziale Krankheit zu verstehen. Ein solches Modellverständnis fördert die Integration der verschiedenen Perspektiven einer Suchterkrankung und erfordert eine biopsychosoziale Bewertung des individuellen Schweregrades einer Suchterkrankung anhand eines multidimensionalen Systems verschiedener Dimensionen, die durch die ASAM-Kriterien ermöglicht wird. Hilfreiche Instrumente zur Messung des Schweregrades einer Suchterkrankung sind z. B. der Addiction Severity Index, der auch in Deutschland bereits seit langem eingeführt ist. Weniger bekannt in Deutschland ist der Recovery Attitude and Treatment Evaluator (RAATE), der die Erkrankungsschwere mit Hilfe von fünf Dimensionen misst. Beide Instrumente basieren auf Patientenerfahrungen aus der Vergangenheit und der gegenwärtigen Situation.

\section{Bewertungsdimensionen}

Neben den Patientencharakterista werden sechs dimensionale Kriterien zur Bewertung der Schwere und Art der Suchterkrankung des Patienten einbezogen und führen in einer Gesamtbewertung zu einer Einteilung in die verschiedenen individuellen Behandlungsstufen. In jeder der Bewertungsdimensionen (Tab. 1) wird die Behandlungsbedürftigkeit einzeln beurteilt.

Mithilfe der ersten Bewertungsdimension „akute Intoxikation mit/ohne Entzugspotenzial“" sollen risikoreiche Konsequenzen für den Patienten bei bestehender Intoxikation vermieden und dem Patienten die Detoxifikation weitestgehend erleichtert wer- 
Tab. 1 Dimensionale Bewertungsdimensionen

\begin{tabular}{|c|c|}
\hline Dimension 1: & akute Intoxikation mit/ohne Entzugspotenzial \\
\hline Dimension 2: & biomedizinischer Behandlungsbedarf/Komplikationen \\
\hline Dimension 3: & $\begin{array}{l}\text { emotionale, kognitive oder Verhaltensbehinderungen/-kompli } \\
\text { kationen }\end{array}$ \\
\hline Dimension 4: & Bereitschaft zur Verhaltensänderung \\
\hline Dimension 5: & $\begin{array}{l}\text { Rückfall, kontinuierlicher Konsum oder kontinuierliches Prob- } \\
\text { lempotenzial }\end{array}$ \\
\hline Dimension 6: & Restabilisierung, Erholung \\
\hline
\end{tabular}

den, um einen bestmöglichen Eintritt in die Behandlung zu gewährleisten. Entsprechende Fragestellungen sind: Welches Risiko ist mit einer akuten Intoxikation verbunden? Sind signifikante Entzugsrisiken aus der Vergangenheit bekannt? Bestehen aktuell Entzugserscheinungen? Ist eine ausreichende Unterstützung bei ambulanter Detoxifikation gewährleistet?

Die zweite und dritte Bewertungsdimension ist auf den medizinischen bzw. psychischen Behandlungsbedarf und daraus resultierende mögliche Komplikationen bei der Behandlung ausgerichtet. Welche Ressourcen stehen dem Patienten bei der Bewältigung von akuten oder chronischen medizinischen bzw. psychischen Krankheiten, die in Wechselwirkung zur Behandlung stehen, zur Verfügung? Bestehen emotionale oder kognitive Probleme im Zusammenhang mit der Suchterkrankung? Bestehen bereits medikamentöse Therapien?

Die vierte Bewertungsdimension fragt nach der Bereitschaft zur Verhaltensänderung in Anlehnung an das bekannte Modell von Proschaska [4]. Bestehen Einwände gegen eine Behandlung oder fühlt sich der Patient zur Therapie gezwungen? Besteht Problemeinsicht oder nur Compliance? Widerstand gegen die Behandlung führt nicht automatisch zum Behandlungsausschluss. Vielmehr ist die Bereitschaft zur Verhaltensänderung ausschlaggebend.

Rückfall, kontinuierlicher Konsum oder kontinuierliches Problempotenzial sind die Bestandteile der fünften Bewertungsdimension. Als Bewertungsgrundlage dienen mögliche Rückfallerfahrungen sowie eine Begutachtung der aktuellen Lebenssituation. Um das Rückfallpotenzial zu bewerten, werden weitere Faktoren berücksichtigt, wie zum Beispiel die aktuelle Wirkung der konsumierten Substanzen. Besitzt der Patient Bewältigungsstrategien, die einen Rückfall vermeiden können? Welche Probleme ergeben sich aus einem Rückfall hinsichtlich der weiteren Behandlung?

In der letzten Bewertungsdimension wird das soziale Umfeld des Patienten beleuchtet. Bestehen im Umfeld Faktoren, die eine Erholung oder Restabilisierung des Patienten unterstützen? Stellen die aktuellen Konstellationen stabilisierende oder destabilisierende Faktoren dar? Besitzt der Patient ein positives Umfeld, Freundschaften, finanzielle Ressourcen oder Voraussetzungen, die eine erfolgreiche Therapie fördern? Besteht für den Patienten die Möglichkeit, an Beratungen zu seiner rechtlichen, beruflichen und sozialen Situation teilzunehmen?
Bei einem Großteil der Patienten sind substanzgebundene Störung und eine weitere psychiatrische Erkrankung miteinander verknüpft. Dabei ist unser Versorgungssystem noch immer inadäquat für die Behandlung komorbider psychiatrischer und substanzabhängiger Patienten eingerichtet. Die Schwierigkeit, einen Patienten in eine therapeutisch angemessene Behandlung zu integrieren, verursacht nicht nur schlechte Behandlungsergebnisse, sondern auch hohe Kosten. Hier ist eine gemeinsame Sprache gefordert, die die Grenzen zwischen den Behandlungssystemen der Psychiatrie und der Suchttherapie öffnet. Ansätze sind in integrierten Behandlungskonzepten $\mathrm{zu}$ finden, die mit flexiblen therapeutischen Behandlungskonzepten den besonderen Bedingungen bei komorbiden Patienten gerecht werden. Allerdings sind existierende integrative Behandlungsangebote bisher vornehmlich für den ambulanten Sektor entwickelt worden. Die Notwendigkeit weiterer Angebote aus gesundheitsökonomischer Sicht steht außer Frage.

Während die erste Version der ASAM-Kriterien ausschließlich auf Behandlungsprogramme zur Suchtbehandlung ausgerichtet war, ist in die aktuelle Version die Behandlung von komorbiden Patienten integriert worden. Patienten mit mäßigen Angststörungen, mäßigen bipolaren Störungen oder mit mäßigen Persönlichkeitsstörungen werden von Patienten mit schweren Begleiterkrankungen wie Schizophrenien unterschieden. Gemäß der Einteilung der Schwere der Begleiterkrankungen werden unterschiedliche Behandlungskonzepte eingesetzt. Bei Patienten mit einer mäßig ausgeprägten Begleiterkrankung steht primär die Behandlung der Suchtstörung im Vordergrund. Patienten mit einer schweren Begleiterkrankung werden in speziellen Behandlungsprogrammen auf beide Erkrankungen hin behandelt. Abhängig vom Schweregrad der Erkrankung ist eine primäre Behandlung auch außerhalb der Behandlungsebene möglich. Bei Stabilisierung des Patienten erfolgt die Begutachtung anhand der Bewertungsdimensionen, wobei die Erkrankung mit dem höheren Schweregrad grundlegend für die Einteilung in eine Behandlungsstufe ist.

\section{Behandlungsstufen}

Entsprechend der ASAM-Bewertung ist eine Suchtbehandlung dann effizient, wenn sie die gesamten Dimensionen einer Suchterkrankung einbezieht und biopsychosozial ausgerichtet ist. Die medizinische Behandlung umfasst die Entgiftung, „Anti-Craving“- und antagonistische Medikation sowie die Substitution. Kognitive Verhaltenstherapie, Motivationstechniken, verständnisorientierte Psychotherapie und Familientherapie sind Verfahren, die in der psychotherapeutischen Behandlung zum Einsatz kommen. Milieutherapeutische Behandlungsverfahren umfassen unter anderem therapeutische Gemeinschaften.

In der zweiten überarbeiteten Version der ASAM-Patientenzuordnungskriterien werden fünf Behandlungsstufen unterschieden (Tab. 2). Die Behandlungsstufen II-IV werden zudem gemäß der Behandlungsintensität in ihrer Ausprägung und Intensität unterteilt, was zu einer höheren Präzision und zu einer 
Tab. 2 Ebenen der Behandlung

\begin{tabular}{|ll}
\hline Level 0,5 & Frühintervention/Prävention \\
\hline Level I & ambulante Behandlung \\
\hline Level II.1 & intensive, ambulante Behandlung \\
\hline Level II.5 & teilstationäre Behandlung, Tagesklinik \\
\hline Level III.1 & betreutes Wohnen mit geringer klinischer Betreuung \\
\hline Level III.3 & betreutes Wohnen mit mittlerer klinischer Betreuung \\
\hline Level III.5 & betreutes Wohnen mit hoher klinischer Betreuung \\
\hline Level III.7 & stationäre Aufnahme unter intensiver, medizinischer Beobachtung \\
\hline Level IV & intensiv-stationäre Behandlung \\
\hline
\end{tabular}

besseren Reliabilität innerhalb den einzelnen Behandlungsstufen führt.

Als Behandlungsstufe 0,5 wurde 1996 die Frühintervention in die ASAM-Patientenzuordnungskriterien übernommen. Frühintervention wird angeboten für Patienten mit einem erhöhten Risiko, eine Suchterkrankung bzw. schädlichen Substanzkonsum zu entwickeln. Neben der individuellen Hilfe werden Risikofaktoren und mögliche Probleme in Zusammenhang mit einem Substanzgebrauch einbezogen. Die Dauer der Frühintervention ist variabel und endet erst, wenn sie bei dem Klienten zur Änderung des Risikoverhaltens führt oder wenn neue Erkenntnisse eine Behandlung auf einer anderen Versorgungsebene nötig machen. Dies kann beispielsweise die nächsthöhere Behandlungsstufe sein.

Die ambulante Behandlung (Behandlungsstufe 1) ist ein strukturiertes Programm mit einer großen Bandbreite an verschiedenen ambulanten Behandlungsstrategien und hat Abstinenz zum Ziel. Suchttherapeutisch geschultes und speziell ausgebildetes, multiprofessionelles Personal (Mediziner, Psychologen, Sozialarbeiter) bewerten die Entwicklung des Patienten und führen die Behandlungen und Beratungen durch, die bis zu 9 Wochenstunden umfassen können. Die qualifizierte Behandlung sollte unter anderem Individual-, Gruppen- und Familientherapie beinhalten, die von Beratungen zu Beruf und Freizeitgestaltung begleitet und von einer Reihe medizinischer Dienste wie Labor und Toxikologie unterstützt wird. Alle Behandlungsangebote stehen im Bedarfsfall 24 Stunden pro Tag zur Verfügung und sind direkt an die höheren Behandlungsstufen angeschlossen.

Die Behandlungsstufe II kennzeichnet eine intensive, ambulante Behandlung, die aber auch bei Bedarf eine teilstationäre Aufnahme einschließt. Kennzeichnend für eine intensive, ambulante Behandlung sind strukturierte, beratende Bildungsprogramme zum Drogengebrauch. Diese Behandlungsstufe ist ausschließlich für weit gehend stabile Patienten mit moderaten Suchtproblemen vorgesehen. Die teilstationäre Behandlung beinhaltet ein intensives, zwanzigstündiges Behandlungsprogramm anhand eines individuellen Behandlungsplans und ist auf Patienten mit somatischer und/oder psychiatrischer Komorbidität ausgerichtet.

Für beide Behandlungsvarianten bestehen neben Angeboten zur psychiatrischen Behandlung Möglichkeiten einer medizinischen medikamentösen Begleittherapie sowie einer 24-h-Krisenintervention, um besonders komorbiden Patienten eine adäquate Versorgung bieten zu können. Die Behandlung wird berufsbeglei- tend angeboten und beinhaltet strukturierte Tages- oder Abendprogramme. Die Patienten haben so die Möglichkeit, die Behandlungskomponenten in ihren Alltag einzubauen.

Patienten der Behandlungsstufe III benötigen eine stabile Umgebung und 24-stündige Betreuung, die eine stationäre Behandlung erforderlich macht, vergleichbar dem betreuten Wohnen. Die Intensität der Behandlung variiert je nach Schweregrad der Erkrankung von einer teilstationären bis zur vollstationären Behandlung. Dabei kommen je nach Bedarf sozio- und psychotherapeutische sowie medizinische Behandlungsverfahren zum Einsatz.

Eine intensive stationäre Akutbehandlung entspricht den Kriterien der Behandlungsstufe IV. Die Behandlung ist auf Patienten mit akuten medizinischen und/oder psychiatrischen Problemen ausgerichtet, die eine schnelle medizinische Behandlung in einem Krankenhaus mit oder ohne psychiatrische Abteilung erfordern. Die täglich 24-stündige medizinische, vollstationäre Überwachung des Patienten erfolgt interdisziplinär durch speziell geschultes medizinisches Personal.

\section{Substitutionstherapie}

Die Opioidsubstitution ist eine im Regelfall ambulant durchgeführte Behandlung für Patienten mit einer bestehenden Opiatabhängigkeit und der Sammelbegriff für eine Vielfalt verschiedener pharmakologischer und nicht-pharmakologischer Behandlungsmodalitäten. Pharmakologisch wird die Substitution entweder mit Methadon oder Buprenorphin durchgeführt. Speziell ausgebildetes Personal begleitet die Therapie mit Beratungsgesprächen zu Risikoerkrankungen wie HIV und Hepatitis C. Dosierung, Behandlungsstufe, Dauer der Behandlung und Behandlungshäufigkeit hängen von den Bedürfnissen des Patienten ab. Die Behandlung sollte in Verbindung mit psychosozialer Betreuung und Psychoedukation durchgeführt werden. Die Opioiderhaltungstherapie wird unabhängig in allen Behandlungsstufen angeboten.

\section{Entgiftung}

Während die früheren Versionen der ASAM-Kriterien die Entgiftung ausschließlich im Rahmen einer stationären Behandlung vorsahen, ist in der aktuellen Version eine Entgiftung in allen Behandlungsstufen möglich, also auch ambulant. Sie verläuft unabhängig von der eigentlichen Behandlung und entspricht in der Intensität den verschiedenen Behandlungsstufen.

\section{Durchfüihrbarkeit, Reliabilität und Validität}

Die Entwicklung der ASAM-Kriterien ist nicht abgeschlossen, vielmehr befinden sie sich in einem stetigen Prozess der Erneuerung und Veränderung ähnlich der Entwicklung des „Diagnostic and Statistical Manual of Mental Disorders" der amerikanischen Gesellschaft für Psychiatrie. In den letzten Jahren waren Durchführbarkeit, Reliabilität und Validität der ASAM-Kriterien Gegenstand verschiedener wissenschaftlicher Studien. 


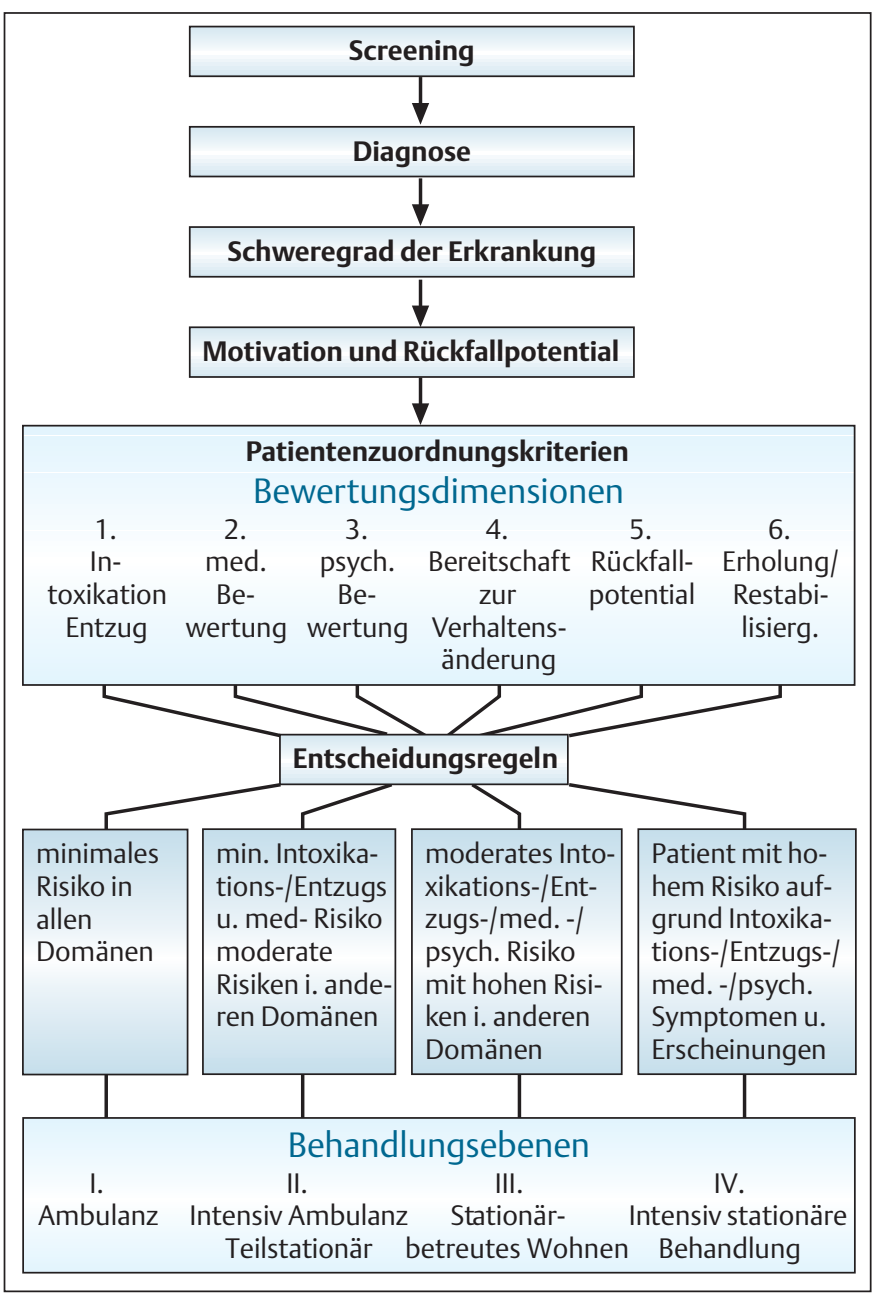

Abb. 1 Hierarchieschema der ASAM-Patientenzuordnungskriterien [5].

Der komplizierte und komplexe hierarchische Aufbau erschwert die Durchführbarkeit einer Patientenzuordnung anhand der ASAM-Kriterien. Zwar führt eine Verkürzung der Kriterien zu Reliabilitätsverlusten, Plough et al. [6] konnten jedoch in einer der ersten Untersuchungen zeigen, dass eine Einteilung und Bewertung der Patienten anhand einer verkürzten Form der Patientenzuordnungskriterien mit einer zu 38\% höheren Wahrscheinlichkeit assoziiert war, die Behandlung innerhalb von 30 Tagen nach der Entgiftung fortzusetzen in Verbindung mit einem signifikant niedrigeren Risiko einer erneuten Entgiftung innerhalb von 90 Tagen. Trotz des komplexen Entscheidungsbaums zeigen die ASAM-Kriterien eine gute Reliabilität (Korrelationskoeffizient $=0.77)[7]$.

Eine andere Studie unter 95 Veteranen konnte den Zusammenhang zwischen einer Patientenzuteilung nach der ersten Version der ASAM-Kriterien und einem reduzierten Krankenhausaufenthalt zeigen [9]. Magura et al. [10] konnten die prädiktive Validität der ersten Version der ASAM-Kriterien nachweisen. Ambulanten Patienten, die einer niedrigeren Behandlungsstufe zugeordnet wurden, als gemäß der Kriterien empfohlen, hatten innerhalb von drei Monaten signifikant häufiger Tage mit Alkoholkonsum als Patienten, die entsprechend den Kriterien zugeordnet wurden. Gleichzeitig zeigten mehrere Untersuchungen, dass eine Versorgung des Patienten in einer höheren Behand- lungsstufe als benötigt keine Vorteile für den Behandlungserfolg hat $[8,9]$. Auch in einer ersten randomisierten kontrollierten Studie konnte Gastfriend die gute, übereinstimmende Validität der ersten Version der ASAM-Kriterien bestätigen [10]. Insgesamt bescheinigen die Untersuchungen den ASAM-Patientenzuordnungskriterien eine gute Durchführbarkeit, Reliabilität und Validität, so dass die Nutzung der ASAM-Kriterien von nationalen Gremien der Vereinigten Statten empfohlen wird.

\section{Möglichkeiten der Implementierung - ein Diskussionsvorschlag}

Angebotene suchttherapeutische Dienstleistungen sind in Deutschland auf mehreren Ebenen diversifiziert und zugleich fragmentiert. Die Diversifikation betrifft die Anbieter sowie die Kostenträger. Anbieter können u. a. Krankenhäuser, niedergelassene Ärzte und Beratungsstellen sein, die Angebote können stationär, teilstationär oder ambulant wahrgenommen werden. Die Kosten können entweder von den Krankenkassen oder den Rentenkassen übernommen bzw. vom Patienten selbst bezahlt werden. Die Diversifikation mag seitens der Anbieter und der Kostenträger folgerichtig sein und spiegelt auch die Bemühungen wider, unterschiedlichen Behandlungsbedürfnissen mit einem differenzierten Behandlungsangebot begegnen zu können. Der Übergang von einem Anbieter zum anderen, die Veränderung der therapeutischen Ebene und die Veränderung der Kostenträgerschaft stellen auf der anderen Seite Sollbruchstellen dar, die einen optimalen Behandlungsverlauf empfindlich unterbrechen können. Die modellhafte Implementierung von Patientenzuordnungskriterien bietet durch ihre patientenorientierte Diagnostik und Ressourcenzuordnung die Möglichkeit, vorhandene Angebote in ihren verschiedenen Facetten zu nutzen, mag jedoch zugleich die Möglichkeit bieten, Sollbruchstellen zu umschiffen. Ein solcher Prozess bedarf jedoch seitens der Leistungsanbieter und der Kostenträger einer weitergehenden Flexibilisierung, da Patientenbehandlungspfade einen eher netzartigen Charakter besitzen und starre Grenzen dem Behandlungsprozess abträglich wären. Das bestehende System ist gekennzeichnet durch Angebote, die sich nicht systematisch auf dem Boden gewachsener Strukturen unter dem Wechselspiel von Anbietern und Kostenträgern entwickelt haben. Als Folge dieser Entwicklung sind Patientenbehandlungsprozesse von Überversorgung auf der einen und Unterversorgung auf der anderen Seite gekennzeichnet. Dies führt zugleich zu verbesserungswürdigen Behandlungspfaden sowie zu fehlgeleitetem Ressourceneinsatz. Die Implementierung von Zuordnungskriterien mag ein Werkzeug zur Systematisierung eines patientenorientierten Behandlungsprozesses sein. Eine modellhafte Umsetzung in einer umgrenzten Region bietet die Möglichkeit zur Evaluation von Zuordnungskriterien in Deutschland. Die Resultate aus den USA ermutigen zu diesem Schritt.

Fazit

Das Versorgungssystem muss in Zukunft interdisziplinäre, multiperspektivische Ansätze in der Krankenversorgung integrieren. Modelle, die optimierte Behandlungsprozesse in Netzwerken und adäquaten Ressourceneinsatz berücksichtigen, haben hier- 
bei Priorität. Die Versorgungsforschung ist gefordert, Struktur, Funktion und Leistungsfähigkeit der Krankenversorgung zu evaluieren und mitzugestalten.

\section{Literatur}

${ }^{1}$ American Society of Addiction Medicine. ASAM PPC-2R: Patient Placement Criteria for the treatment of Substance-Related Disorders, second Edition revised. Chevy Chase, Maryland, USA 2001

2 Donovan DM. Assessment of addictive behaviors: implications of an emerging biopsychosocial model. In: Donovan DM and Marlatt GA (Hrsg). Assessment of Addictive Behavior. New York: Guilford Press, 1988

${ }^{3}$ Wallace J. The new disease model of alcoholism. Western Journal of Medicine 1990; 152: 502 - 505

${ }^{4}$ Proschaska JO, Norcross JC. Stages of change. Psychotherapy 2001; 38: $443-448$
${ }^{5}$ Gastfriend DR, McLellan AT. Treatment matching: Theoretic basis and practical implications. Medical Clinics of North America 1997; 81: 945-966

${ }^{6}$ Plough A, Shirley L, Zaremba N et al. CSAT Target Cities Demonstration Final Evaluation Report. Boston, MA: Office for Treatment Improvement, USA, 1996

${ }^{7}$ Baker SL, Gastfriend DR. Reliability of Multidimensional Substance Abuse Treatment Matching: Implementing the ASAM Patient Placement Criteria (in press).

${ }^{8}$ Sharon E, Krebs C, Turner W et al. Predictive Validity of the ASAM Patient Placement Criteria for Hospital Utilization (in press).

${ }^{9}$ Magura S, Staines G, Kosanke N et al. Predictive validity of the ASAM Patient Placement Criteria: Outcomes for patients naturalistically matched and mismatched to Levels of Care. Journal of Substance Abuse Treatment 2003; 29: $203-218$

${ }^{10}$ Gastfriend DR. ASAM Anual Medical-Scientific Meeting. Los Angeles CA 2000

${ }^{11}$ Krausz M. Platzierungskriterien für die Suchttherpie. AMDP-Tagung: Erfolgsmessung und Verlaufsdiagnostik. Hamburg 2003 